Rev. Tbe. 65, 718 (1952). - 16. Henning, N., Klinische Laboratoriumsdiagnostik, S. 11, 16, 21, 67, 81, 84, 85, 144 und 409. Urban \& Schwarzenberg, München und Berlin (1960). - 17. Frenci, J. E. und B. J. Morris, Physiology 138, 326 (1957). 18. Sherber, D. A. und M. M. Levites, J. A. M. A. 152, 682 (1953). - 19. Zilversmit, D. B., C. Entenmann und I. L. CihatKorr, J. biol. Chemistry 176, 209 (1948). - 20. CoHN, E. J., L.E. Strong, W. L. Hughes, D. J. Mulford, J. N. Ashworth, M. Melin und H. L. TAYlor, J. Amer. chem. Soc. 68, 459 (1946). 21. Oncley, J. L., F. R. N. Gurd und M. Melin, J. Amer. chem. Soc. 72, 458 (1950). - 22. Dunhiam, L. J. und A. Brunschwig, Arch. Surg. 48, 395 (1944). - 23. FreEMAN, L. W. und V. JohNson, Amer. J. Physiol. 124, 466 (1938). - 24. Johnson, V. und
L. W. Freeman, Amer. J. Physiol. 130, 723 (1940). - 25. LongNini, J., L. W. Freeman und V. Johinson, Federat. Proc. 1, 51 (1942). - 26. Johnson, V., J. Longnini und L. W. FreEman, Science 97, 400 (1943). - 27. Josepirs, H. W., L. E. Holt jr., H. C. Tidwell und CH. N. KadjrDr, J. Clin. Invest. 17, 532 (1938). 28. Kibbin Mac, J. M., A. Pope, S. Thayer, R. M. Ferry jr. und F. J. Stare, J. Laborat. Clin. Med. S. Louis 30, 488 (1945). 29. Kibbin Mac, J. M., J. Clin. Invest. 25, 679 (1946). - 30. Meng, H. C., Parenteral alimentation of carbohydrate and fat. Master's Thesis, Northwestern Universitiy Medical School (1946). - 31. Meng, H. C. und L. W. Freenan, J. Laborat. Clin. Med. S. Louis 33,689 (1948).

\title{
Aldosteronfraktionen im Plasma hepatektomierter Hunde')
}

\author{
Von \\ M. ManN, W. Siegenthaler, K. KRAMpf und E. ZINGG \\ Aus der Medizinischen Universitäts-Poliklinik Zürich (Direktor: Prof. R. Hegglin) und dem Experimentallabor der \\ Cbirurgischen Universitätsklinik A, Zïrich (Direktor: Prof. A. Senning)
}

(Der Schriftleitung zugegangen am 21. Februar 1964)

\begin{abstract}
Nach Injektion von 1,2- ${ }^{3} \mathrm{H}-\mathrm{d}-$ Aldosteron wurden im Plasma von 3 normalen und von 3 hepatektomierten Hunden dic prozentualen Anteile der injizierten Radioaktivität bestimmt, die als freies Aldosteron, als 3-Oxo-Konjugat und als $\beta$-Glukuronidase labile $\mathrm{pH}$ 5-Fraktion, in der das Tetrahydroaldosteronglukuronid enthalten ist, vorlagen.

Das freie Aldosteron ( $\mathrm{pH} 6$ ) ist im Plasma hepatektomierter Hunde in größerer Konzentration vorhanden als im Plasma normaler Hunde. Die pH 5-Fraktion mit dem Tetrahydroaldosteronglukuronid dagegen liegt im Plasma hepatektomierter Hunde in geringerer Konzentration vor als im Plasma normaler Hunde. Die äußerst geringe Konzentration des 3-Oxo-Konjugates sowohl im Plasma hepatektomierter als auch normaler Hunde weist bei gleichzeitigem Vorkommen erheblicher Mengen im Urin der Tiere auf eine extrem hohe renale Clearance dieses Metaboliten hin. Dies kann als Hinweis für eine möglicherweise in der Niere stattfindende Transformation von Aldosteron in das 3-Oxo-Konjugat aufgefaßt werden.
\end{abstract}

\begin{abstract}
After i. v. Injection of $1,2-{ }^{3} \mathrm{H}-\mathrm{d}-$-Aldosterone in 3 normal and 3 hepatectomised dogs the percentage of injected radioactivity of the 3 different aldosterone fractions, free aldosterone $(\mathrm{pH} 6), 3$-oxo-conjugate $\left(\mathrm{pH}_{1}\right)$ and tetrahydroaldosteroneglucuronide $\left(\mathrm{pH}_{5}\right)$ in the plasma were determined.

The free aldosterone $\left(\mathrm{pH}_{6}\right.$-fraction) in the plasma of hepatectomised dogs reaches a higher concentration than in the plasma of normal dogs. The $\mathrm{pH}_{5}$-fraction in the plasma of hepatectomised dogs has been found in a smaller amount than in non hepatectomised animals. However in the plasma of normal and hepatectomised dogs the concentration of the 3-oxo-conjugate is very small while in the urine of the same dogs a remarkable quantity of this metabolite was found. This high rate of clearance of the 3-oxo-conjugate may be considered as a suggestion for a possible transformation into this metabolite in the kidneys.
\end{abstract}

Auf Grund der im Urin von hepatektomierten Hunden nach Injektion von $1,2-{ }^{3} \mathrm{H}-\mathrm{d}$-Aldosteron gefundenen radioaktiven Aldosteronmetabolite konnten wir (1) zei= gen, daß Aldosteron bis zu einem gewissen Grade auch außerhalb der Leber metabolisiert werden kann. Dabei kann die Umwandlung von Aldosteron zum 3:OxoKonjugat weitgehend extrahepatisch stattfinden, während diejenige zum Tetrahydroaldosteronglukuronid nur

1) Diese Arbeit wurde durch die Hilfe des Schweizerischen Nationalfonds zur Förderung der wissenschaftlichen Forschung und der Stiftung für wissenschaftliche Forschung an der Universität Zürich ermöglicht. in bescheidenem Ausmaß extrahepatisch erfolgen kann. Die folgenden Ausführungen beziehen sich auf das Verhalten der verschiedenen Aldosteronfraktionen im Plasma hepatektomierter Hunde. Dabei stellte sich die Frage, ob nach Injektion von 1,2-3 $\mathrm{H}$-d-Aldosteron auch im Plasma radioaktive Aldosteronmetabolite, die somit extrahepatisch in die inaktiven Ausscheidungsformen umgewandelt würden, nachweisbar sind.

\section{Methode}

Es handelt sich um dieselbe Versuchsanordnung, die auch für den Nachweis der verschiedenen Aldosteron- 
fraktionen im Urin angewendet worden ist (1). Nach der Hepatektomie und der anschließenden i. v.-Injektion

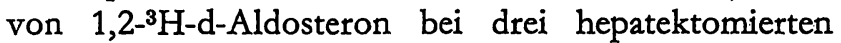
Hunden wurden in regelmäßigen Abständen $50-100 \mathrm{ml}$ Blut aus der A. femoralis entnommen und sofort heparinisiert. Bei drei nicht hepatektomierten, als Kontrollen dienenden Hunden erfolgte ein sonst analoges Vorgehen nach i. v.-Injektion von $1,2-{ }^{3} \mathrm{H}$-d-Aldosteron. Nachdem das Blut zentrifugiert und das Plasma abpipettiert worden war, wurde letzteres bis zur Aufarbeitung im Tiefkühlschrank aufbewahrt.

Vor der Auftrennung in die verschiedenen Fraktionen wurden die Gesamtimpulse im Plasma bestimmt. Hierfür wurden zu $0,2 \mathrm{~m} l$ Plasma $3 \mathrm{~m} l$ absoluter Alkohol und $10 \mathrm{~m} l$ Toluol-Szintillationsflüssigkeit zugesetzt. Der "Quenching-Effekt", der $30-40 \%$ betrug, wurde durch gleichzeitige Zählung eines Standards berücksichtigt. - Mittels Präzipitation mit absolutem Alkohol und Zentrifugation wurde das die nachfolgenden Untersuchungen störende Eiweiß abgetrennt, während sich gleichzeitig eine allfällig vorhandene Eiweißbindung des Aldosterons löste. Mittels destilliertem Wasser wurde das von Eiweiß befreite Plasma sodann auf ein Volumen von $20 \mathrm{~m} l$ gebracht. Die weitere Verarbeitung (Zufuhr von Tracer, Extraktion, Isolierung mittels Chromatographien, Bestimmung der Radioaktivität und schließlich Berechnung) erfolgte entsprechend dem für die Urinuntersuchungen angegebenen Verfahren (1).

\section{Ergebnisse}

Die Konzentration der im Plasma vorhandenen Totalaktivität hielt sich bei den 3 hepatektomierten Hunden in der Zeit vom Beginn der 2. bis zum Abschluß der 6. Stunde post injectionem von radioaktivem Aldosteron beinahe auf dem gleichen Niveau. Bei den nicht hepatektomierten Hunden dagegen fand in dieser Zeit ein deutlicher Abfall der im Plasma vorhandenen Konzentration der Radioaktivität statt (Abb. 1).

Die Anzahl Impulse der verschiedenen Aldosteronfraktionen, die in je $100 \mathrm{ml}$ Plasma, 2 bzw. 4 Stdn. nach der

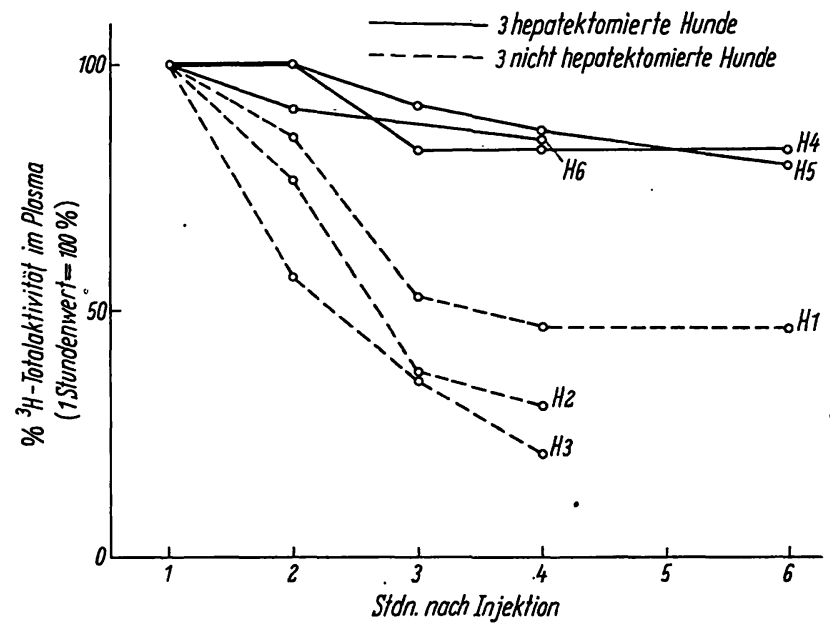

Abb. 1

Verlangsamter Schwund der Totalaktivität im Plasma nach Injektion von $1,2-{ }^{3} \mathrm{H}$-d-Aldosteron bei 3 hepatektomierten Hunden im Vergleich zu 3 nicht hepatektomierten Tieren
Injektion von $1,2-{ }^{3} \mathrm{H}-\mathrm{d}$-Aldosteron gefunden wurden, sind in $\%$ der injizierten Impulse ausgedrückt aus Tabelle 1 ersichtlich.

Tab. 1

Anzahl Impulse der verschiedenen Aldosteronfraktionen in je $100 \mathrm{ml}$ Plasma von 3 hepatektomierten und 3 nicht hepatektomierten Hunden, ausgedrückt in $\%$ der injizierten Dosis, 2 bzw. 4 Stdn. nach Injektion von $1,2-{ }^{3} \mathrm{H}$-d-Aldosteton

\begin{tabular}{|c|c|c|c|c|c|c|}
\hline \multirow{4}{*}{$\begin{array}{l}\text { nicht hepa- } \\
\text { tektomierte } \\
\text { Hunde }\end{array}$} & \multirow{4}{*}{$\begin{array}{l}\text { Hund } 1 \\
\text { Hund } 2 \\
\text { Hund } 3\end{array}$} & $\begin{array}{l}\text { Freies } \\
\text { Aldosteron }\end{array}$ & \multicolumn{2}{|c|}{$\begin{array}{l}\text { 3-Oxo- } \\
\text { Konjugat }\end{array}$} & \multicolumn{2}{|c|}{$\begin{array}{c}\text { pH 5- } \\
\text { Metabolit }\end{array}$} \\
\hline & & 0,49 & 0,04 & 0,02 & 1,01 & \\
\hline & & 0,40 & 0,02 & $<0,01$ & 0,35 & \\
\hline & & 0,50 & $<0,01$ & 0,02 & 0,15 & \\
\hline & \multicolumn{2}{|c|}{ Mittelwerte $\quad 0,51$} & \multicolumn{2}{|c|}{0,02} & \multicolumn{2}{|c|}{0,43} \\
\hline bepatekto = & (Hund 4 1,45 & 1,19 & 0,06 & 0,02 & 0,49 & 0,4 \\
\hline mierte & $\{$ Hund $5 \quad 1,81$ & 0,29 & 0,03 & $<0,01$ & 0,32 & 0,13 \\
\hline \multirow[t]{2}{*}{ Hunde } & Hund $6 \quad 2,70$ & 0,54 & 0,02 & 0,07 & 0,11 & \\
\hline & Mittelwerte 1 , & te 1,33 & \multicolumn{2}{|c|}{0,03} & \multicolumn{2}{|c|}{0,30} \\
\hline
\end{tabular}

Die Mittelwerte aus den bei den 3 nicht hepatektomierten Hunden bestimmten 2- und 4-Stdn.-Fraktionen ergeben für das freie Aldosteron $0,51 \%$, für das 3-OxoKonjugat $0,02 \%$ und für die $\mathrm{pH}_{5}$-Fraktion $0,43 \%$. Die entsprechenden Mittelwerte bei den 3 hepatektomierten Hunden dagegen betragen für das freie Aldosteron $1,33 \%$, für das $3-O x o-K o n j u g a t ~ 0,03 \%$ und für den $\mathrm{pH}_{5}$-Metaboliten $0,30 \%$ (Tab. 1, Abb. 2).

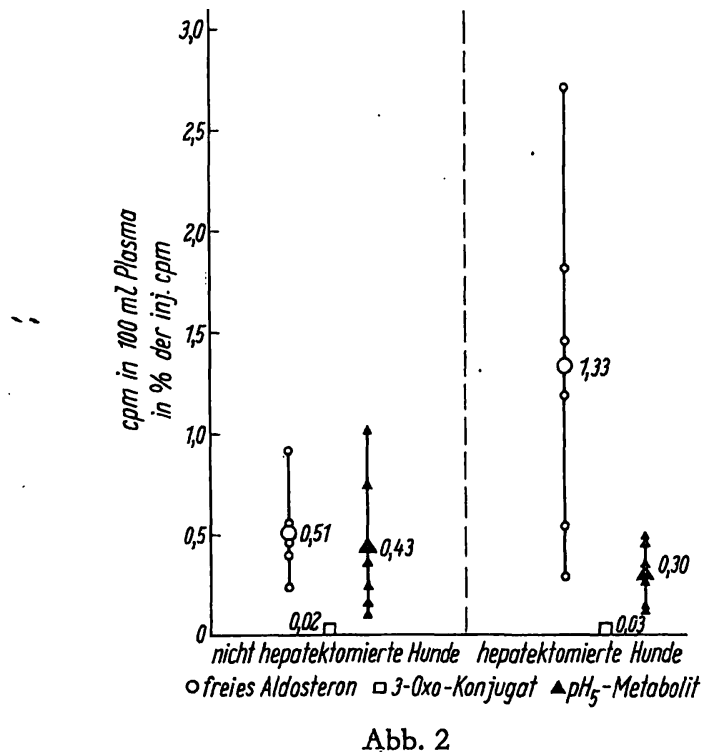

Mittelwerte und Einzelwerte der 3 Aldosteronfraktionen im Plasma von 3 hepatektomierten und 3 nicht hepatektomierten Hunden 2 und 4 Stunden nach Injektion von $1,2-{ }^{3} \mathrm{H}$-d-Aldosteron

\section{Diskussion}

Nach Injektion von 1,2-3 H-d-Aldosteron, das denselben Stoffwechsel wie endogenes Aldosteron durchmacht (2), nimmt die Totalaktivität im Plasma hepatektomierter Hunde im Verlauf von 1-6 Stdn. kaum ab, während bei nicht hepatektomierten Hunden ein deutlicher Abfall zu beobachten ist (Abb. 1). Dies ist damit zu erklären, daß die Abwanderung des Aldosterons aus dem 
Plasma nicht allein die Folge der Ausscheidung, sondern besonders auch der Verteilung im Organismus ist. Dabei gelangt normalerweise ein beträchtlicher Anteil des Aldosterons in die Leber. Fehlt die Leber, so ist der Plasmaschwund erheblich verlangsamt (Abb. 1).

2. bzw. $4 \mathrm{Stdn}$. nach Injektion von $1,2-{ }^{3} \mathrm{H}-\mathrm{d}-A 1 d o-$ steron liegt das freie, d. h. nicht metabolisierte Aldosteron ( $\mathrm{pH}$ 6-Fraktion) im Plasma hepatektomierter Hunde im Mittel in höherer Konzentration vor als bei nicht hepatektomierten Tieren (Tab. 1, Abb. 2).

Die $\mathrm{pH}$ 5-Fraktion, in der das Tetrabydroaldosteronglukuronid enthalten ist, ist dagegen bei den hepatektomierten Tieren im Mittel in etwas geringerer Konzentration im Plasma nachweisbar (Tab. 1, Abb. 2). Dies bestätigt unsere bei den Urinuntersuchungen gemachten Beobachtungen, wonach Aldosteron auch extrahepatisch bis zu einem gewissen Grade in diesen Metaboliten umgewandelt werden kann (1).

Das 3-Oxo-Konjugat ( $\mathrm{pH}$ 1-Fraktion) ist dagegen im Plasma hepatektomierter und nicht hepatektomierter Hunde nur in Spuren vorhanden. Wegen dieser geringen Konzentration lassen Plasmauntersuchungen daher kei- nen eindeutigen Schluß hinsichtlich einer möglichen extrahepatischen Umwandlung von Aldosteron in diesen Metaboliten zu. Unsere im Urin hepatektomierter Hunde durchgeführten Untersuchungen zeigen allerdings deutlich, daß die Metabolisierung des Aldosterons zum 3-Oxo-Konjugat weitgehend extrahepatisch erfolgen kann (1). Die erheblichen Unterschiede in der Konzentration dieses Metaboliten in Plasma und Urin $(1,3)$ sprechen für eine sehr hohe renale Clearance. Beim Menschen konnten wir zeigen, da $\beta$ die Clearance des 3-Oxo-Konjugates bedeutend höher liegt als der renale Plasmadurchfluß, was als strenger Hinweis auf eine in der Niere stattfindende Metabolisierung von Aldosteron zum 3-Oxo-Konjugat aufzufassen ist $(3,4)$. SANDOR und LANTHIER (5) konnten mittels Inkubation von Nierengewebsschnitten mit ${ }^{14} \mathrm{C}$-d-Aldosteron zeigen, $\mathrm{da} \beta$ in der Niere eine Konjugation von Aldosteron in die Glukuronidfraktion einerseits und in die säurelabile Fraktion (3-Oxo-Konjugat) andererseits möglich ist. Weitere Untersuchungen sind notwendig, um Auskunft über das Ausmaß dieser Metabolisierung in der Niere zu erhalten.

\title{
Literatur
}

1. ManN, M., W. Siegenthaler; K. Krampr und E. ZingG, Klin. Wschr. 42, 319 (1964). - 2. Ulick, S., J. biol. Chemistry 236, 680 (1961). - 3. Siegenthaler, W., R. Peterson und G. Frimpter, in: An International Symposium on Aldosterone, Blackwell Scientific Publications Ltd., Oxford, im Druck (1964). -
4. Gfeller, J., Die renale Clearance von freiem Aldosteron vor und nach Applikation eines Aldosteronantagonisten. Inaug. Diss. Zürich (1964). - 5. SANDor, T. und A. Lanthier, Acta endocr., K'hvn. 39, 87 (1962).

\section{pH-Abhängigkeit der Wirkung von Butazolidin und Tanderil}

\author{
Von \\ H. Holzer und B. Ulrich \\ Aus dem Biochemischen Institut der Universität Freiburg im Breisgau (Direktor: Prof. Dr. H. Holzer) \\ (Der Schriftleitung zugegangen am 6. April 1964)

\begin{abstract}
„Tanderil““ (1-Phenyl-2-(p-hydroxyphenyl)-3,5-dioxo-4-n-butyl-pyrazolidin) hemmt ebenso wie „Butazolidin“ (1,2Diphenyl-3,5-dioxo-4-n-butyl-pyrazolidin) in schwach saurem Milieu die Glykolyse von Ascites-Zellen um ein Vielfaches stärker als im neutralen Milieu. Da entzündete Gewebe und ihre Umgebung schwach sauer sind, könnte die Spezifität der Entzündungs-hemmenden Wirkung von Butażolidin und Tanderil mit den pH-abhängigen Permeationseigenschaften zusammenhängen.

„Tanderil“ (1-phenyl-2-(p-hydroxyphenyl)-3,5-dioxo-4-n-butyl-pyrazolidine) and „butazolidine“ (1,2-diphenyl-3,5dioxo-4-n-butyl-pyrazolidine) inhibit glycolysis in ascites cells far more. strongly in a weakly acidic medium than in a neutral medium. Since the inflamed tissues are weakly acidic, the specificity of the anti-inflammatory action of butazolidine and tanderil may be due to their $\mathrm{pH}$-dependent permeation properties.
\end{abstract}

Wie wir in früheren Versuchen fanden (1), ist die Hemmung der Atmung und Glykose von EhrlichAscites-Zellen durch „Butazolidin“ (1,2-Diphenyl-3,5dioxo-4-n-butyl-pyrazolidin) vom $\mathrm{pH}$ abhängig. Bei $\mathrm{pH}=6$ findet man eine mehr als 10 fach stärkere Glykolysehemmung als bei $\mathrm{pH}=7,2$. $\mathrm{Da}$ entzündete Gewebe ein saureres $\mathrm{pH}$ aufweisen als Normalgewebe, ist es möglich, daß die stärkere Wirksamkeit des Butazolidin in saurerem Milieu an der Spezifität der Entzündungshemmung beteiligt ist. Sehr wahrscheinlich ist es zwar nicht eine Hemmung der Atmung oder der Glykolyse, die die Entzündungshemmung bewirkt, jedoch können 